\section{RESULTS OF EXTRACORPOREAL MEMBRANE OXYGENATION IN NEONATES WITH SEPSIS}

\section{The Extracorporeal Life Support Organization experience}

From the Department of Surgery (Division of Thoracic and Cardiovascular Surgery), University of Texas Southwestern Medical Center, Dallas, Tex., and the University of Michigan, Ann Arbor, Mich.

Read at the Seventy-fourth Annual Meeting of The American Association for Thoracic Surgery, New York, N.Y., April 24-27, 1994.

Address for reprints: Dan M. Meyer, MD, Department of Surgery, Division of Thoracic and Cardiovascular Surgery, University of Texas Southwestern Medical Center, 5323 Harry Hines Blvd., Dallas, TX 75235-8879.

Copyright 1995 by Mosby-Year Book, Inc. $0022-5223 / 95 \$ 3.00+0 \quad \mathbf{1 2} / 6 / 61486$
Use of extracorporeal membrane oxygenation for treatment of respiratory failure caused by sepsis is controversial because of concerns over survival benefit and hemorrhage-related complications. To evaluate the impact of the primary diagnosis of sepsis on outcome, we reviewed data from 6853 neonates in the Extracorporeal Life Support Organization Registry and defined two groups: group 1 ( $n=1060$ ), all patients undergoing extracorporeal membrane oxygenation with a primary diagnosis of sepsis; group $2(n=5793)$, those with any other primary diagnosis. A multivariate logistic regression analysis that considered 15 variables present before extracorporeal membrane oxygenation (including age, sex, birth weight, prior cardiopulmonary arrest, arterial blood gas results, and ventilator settings) was used to compare outcomes between groups. Survival was not different between the two groups $(77 \%$, group $1 ; 82 \%$, group $2 ; p=0.2480$ ), although lung recovery was less frequent in the patients with sepsis $(p=0.0185)$. Group 1 had a higher incidence of complications including seizures (odds ratio $1.446, p=\mathbf{0 . 0 3 4 6}$ ), cerebral infarct or hemorrhage $(2.310, p=0.0001)$, need for dialysis $(1.478, p=0.0131)$, hypernatremia (2.089, $p=0.0019)$, hyperbilirubinemia $(2.423, p=0.0001)$, and dobutamine use $(1.918, p=0.0001)$. Neonates with sepsis are more likely to have neurologic, renal, and metabolic complications from extracorporeal membrane oxygenation but may still achieve a survival benefit equivalent to those without sepsis. From these data, extracorporeal membrane oxygenation should not be withheld from neonates solely on the basis of sepsis. Rather, management strategies should focus on limiting the incidence or severity of the common complications. (J THORaC Cardiovasc Surg 1995;109:419-27)

Dan M. Meyer, MD (by invitation), Michael E. Jessen, MD (by invitation), Dallas, Tex, and the Extracorporeal Life Support Organization, Ann Arbor, Mich. Sponsored by W. Steves Ring, MD, Dallas, Tex.
$\mathrm{T}_{\mathrm{a}}^{\mathrm{h}}$ he development of sepsis in the neonate carries a high mortality because of the potential for rapid progression to septic shock with its resultant physiologic changes. ${ }^{1,2}$ With group B streptococcal sepsis, the most common form of neonatal sepsis in the United States, the acute syndrome is characterized by respiratory distress, hypoxemia, vascular collapse, disseminated intravascular coagulation, and the development of a syndrome of persistent pulmonary hypertension with right-to-left shunting. ${ }^{3}$ Before the introduction of extracorporeal membrane oxygenation (ECMO), management of this condition was limited to medical therapy based on organism-targeted antibiotic coverage, aggressive ventilator management, and vasopressor or inotropic support. Results of conventional therapy were poor when the course was complicated by pulmonary dysfunction, with predicted mortality rates approaching $80 \%{ }^{4}$ As a result, some clinicians have applied ECMO to the management of the infant with sepsis, despite controversy regarding its use in septic states.

Since its introduction in $1975,{ }^{5}$ ECMO has become the standard of care for neonates with severe respiratory failure resulting from meconium aspiration syndrome, ${ }^{6,7}$ persistent pulmonary hypertension of the newborn, ${ }^{8}$ and congenital diaphragmatic hernia with persistent fetal circulation..$^{9}$ Currently, the application of ECMO in neonatal sepsis, although widely performed, is less accepted because of concerns over survival benefit and hemorrhagerelated morbidity. ${ }^{10}$ Other standard therapeutic options in the seriously ill neonate are largely ineffective, however. To date, available reports have involved small numbers of patients in whom ECMO was used for the management of sepsis, limiting the ability to fully analyze the benefits of this modality when other variables are considered. This study was undertaken to critically evaluate the effectiveness of ECMO in neonatal sepsis as reported to the Extra- 


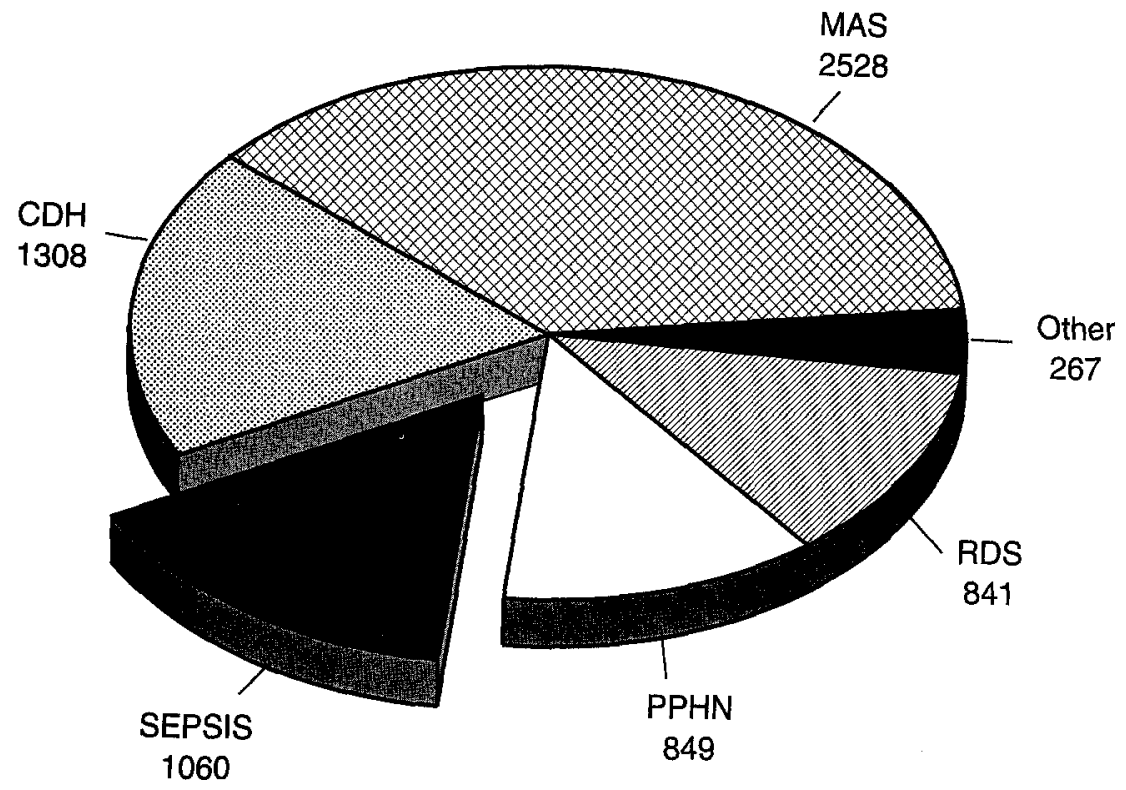

Fig. 1. Frequency distribution of 6856 neonates subjected to ECMO. MAS, Meconium aspiration syndrome; $C D H$, congenital diaphragmatic hernia with persistent fetal circulation; $P P H N$, persistent pulmonary hypertension of the neonate; $R D S$, respiratory distress syndrome.

corporeal Life Support Organization (ELSO) Registry.

\section{Methods}

Patients. This study consisted of 6856 neonates (aged 14 days or less) supported by ECMO between November 1987 and June 1993 and entered in the ELSO Registry. The primary indication for ECMO was available for all but three of the patients, and the frequency of each indication is presented in Fig. 1. For analysis, the data were divided into two groups. Group 1 consisted of all patients with a primary diagnosis of neonatal sepsis $(n=$ 1060 ), and group 2 included those patients with any other primary indication $(n=5793)$. Of note, 127 members of group 1 also carried a secondary diagnosis of sepsis or pneumonia or both, and 639 members of group 2 carried either secondary diagnosis. The presence of either of these secondary diagnoses was not considered in the analysis.

Outcome variables. Outcome variables studied included survival (at time of reporting), recovery (of lung function leading to discontinuation of ECMO), and duration of ECMO support (in hours). Moreover, 51 ECMO complications were available for analysis. Additional variables used in the comparison were the volume of packed red blood cells, fresh frozen plasma, and platelets administered during the peri-ECMO period and the use of drugs (vasoactive agents, bicarbonate, and surfactant) in the patients supported by ECMO. The 68 outcome variables are listed in Appendix A.

Pre-ECMO factors. In addition to the primary indication for ECMO, 15 variables were used in the evaluation as predictors of outcome from ECMO. These variables included patient age at the time of ECMO, sex, birth weight, and a history of cardiopulmonary arrest before ECMO. Also, the results of the most recent arterial blood gas analysis before ECMO, the ventilator parameters of the patient immediately before initiation of ECMO, and the most recent oxygenation index (calculated as [inspired oxygen fraction $\times$ mean airway pressure $\times 100]$ /arterial oxygen tension) were used in the analysis. The type of ECMO was not included because $89 \%$ underwent ECMO via a venoarterial technique. The pre-ECMO variables used are listed in Appendix B.

Statistical analysis. Data were analyzed by means of the SAS software package (SAS Institute, Cary, N.C.). A univariate analysis of all outcome variables was performed initially to compare results between groups 1 and 2 . A $\chi^{2}$ analysis was used for discrete variables, with a $p$ value less than 0.05 by two-tailed Fisher's exact test considered significant. For continuous variables, Student's $t$ test with Welch's approximation for unequal variances was used to compare results between the two groups. This analysis was also considered significant at a $p$ value of less than 0.05 .

We used additional tests to account for interactions between pre-ECMO variables. For dichotomous outcome variables, a multivariate stepwise logistic regression analysis for each outcome event was performed. The group variable plus all variables listed in Appendix B were candidates for entry into the model. For each resulting model, the group variable was always included, whereas only those remaining variables that met the 0.05 significance level were entered. For each element in the model, a parameter estimate was calculated from which a $p$ value and an odds ratio for the variable were derived. From 
Volume 109, Number 3

Table IA. Outcome variables found to occur significantly more frequently $(p<0.05)$ in patients with a primary diagnosis of sepsis as determined by univariate analysis

\begin{tabular}{|c|c|c|c|}
\hline Variable & Group 1 & Group 2 & $p$ Value \\
\hline Brain death $(\%)$ & 2.3 & 0.9 & 0.0003 \\
\hline Seizures $(\%)$ & 17.1 & 12.1 & 0.0001 \\
\hline Infarct or hemorrhage by head ultrasound $(\%)$ & 24.9 & 10.9 & 0.0001 \\
\hline Infarct or hemorrhage by CT/MRI (\%) & 6.5 & 3.0 & 0.0001 \\
\hline Other neurologic problem $(\%)$ & 5.5 & 3.6 & 0.0042 \\
\hline Creatinine $>1.5 \mathrm{mg} / \mathrm{dl}(\%)$ & 14.1 & 9.7 & 0.0001 \\
\hline Dialysis/hemofiltration (\%) & 18.6 & 13.0 & 0.0001 \\
\hline Myocardial stunning (\%) & 8.2 & 6.2 & 0.0206 \\
\hline Symptomatic PDA (\%) & 5.0 & 3.3 & 0.0112 \\
\hline Culture-proven secondary infection $(\%)$ & 13.2 & 4.2 & 0.0001 \\
\hline Suspected secondary infection, elevated WBC count (\%) & 4.2 & 1.8 & 0.0001 \\
\hline Other infectious problem $(\%)$ & 1.9 & 1.0 & 0.0182 \\
\hline $\mathrm{Na}^{+}>150 \mathrm{mEq} / \mathrm{L}(\%)$ & 6.5 & 2.6 & 0.0001 \\
\hline $\mathrm{Ca}^{++}<6 \mathrm{mg} / \mathrm{dl}(\%)$ & 3.3 & 1.5 & 0.0003 \\
\hline Glucose $<40 \mathrm{mg} / \mathrm{dl}(\%)$ & 3.3 & 2.0 & 0.0104 \\
\hline Glucose $>240 \mathrm{mg} / \mathrm{dl}(\%)$ & 3.5 & 2.1 & 0.0073 \\
\hline $\mathrm{pH}<7.20(\%)$ & 4.5 & 1.6 & 0.0001 \\
\hline Hyperbilirubinemia (\%) & 13.6 & 5.8 & 0.0001 \\
\hline Dopamine use (\%) & 81.6 & 74.0 & 0.0001 \\
\hline Dobutamine use (\%) & 60.8 & 46.5 & 0.0001 \\
\hline Epinephrine use (\%) & 18.7 & 9.9 & 0.0001 \\
\hline Bicarbonate use (\%) & 54.7 & 35.9 & 0.0001 \\
\hline Surfactant use (\%) & 14.7 & 9.2 & 0.0001 \\
\hline
\end{tabular}

Values are expressed as percentage occurrence for each group. $C T$, Computed tomography; $M R I$, magnetic resonance imaging; $P D A$, patent ductus arteriosus; $W B C$, white blood cell.

each model, a $p$ value of less than 0.05 was considered significant for the group variable.

For continuous outcome variables, a stepwise multiple regression analysis was performed. All candidate variables were the same (Appendix B). Parameter estimates for the group variable in the resulting models were calculated and statistical significance was defined at the 0.05 level.

\section{Results}

Overall results. Survival was documented in $81.3 \%$ of the entire group of 6856 neonates. Lung recovery leading to separation from ECMO occurred in $85.3 \%$ of the total. Although any individual ECMO complication occurred infrequently, at least one complication occurred in 5310 patients (77.5\%) and more than one complication was documented in 3095 (54.9\%).

Univariate analysis. A univariate analysis of each outcome variable was undertaken to compare the 1060 neonates with a primary diagnosis of sepsis against the remaining 5793 patients. The exact cause of sepsis was not available for all patients, but $74 \%$ of those with documentation of source had group B streptococcal sepsis. No other organism represented more than $9 \%$ of group 1. Outcome variables that were found to be significantly different are listed in
Tables IA and IB along with their mean values or frequency of occurrence as appropriate. Of note, survival was significantly worse in the septic group $(p=0.0002)$ and recovery of lung function was also less likely $(p=0.0001)$ by this analysis. Patients undergoing ECMO as salvage from sepsis were also more likely to have severe neurologic, renal, and metabolic problems (see Table IA). Inotropic agents and bicarbonate were more frequently administered. Only the development of pneumothorax and mechanical bleeding complications were statistically more frequent in the nonseptic group (group 2). Administration of red blood cells and fresh frozen plasma was also increased in group 2. The duration of ECMO was longer in group $2(p=0.0006)$.

Multivariate analysis. To account for potential interactions between pre-ECMO variables, we performed a stepwise regression for each variable as described earlier. Outcome variables that were found to be significantly different between the two groups at a $p$ value of 0.05 are listed in Tables IIA and IIB. As expected, fewer outcomes were significantly different between groups by this analysis.

In particular, survival was not predicted by sepsis $(p=0.2480)$. Variables that did predict survival 
Table IB. Outcome variables found to occur significantly less frequently $(p<0.05)$ in patients with a primary diagnosis of sepsis as determined by univariate analysis

\begin{tabular}{|c|c|c|c|}
\hline Variable & Group 1 & Group 2 & p Value \\
\hline Survival $(\%)$ & 77.0 & 82.1 & 0.0002 \\
\hline Lung recovery $(\%)$ & 75.9 & 85.3 & 0.0001 \\
\hline Duration of ECMO (hr) & $133 \pm 86$ & $143 \pm 90$ & 0.0006 \\
\hline Bleeding at cannula site (\%) & 3.9 & 6.5 & 0.0012 \\
\hline Bleeding at other surgical site (\%) & 2.5 & 7.2 & 0.0001 \\
\hline Pneumothorax necessitating treatment $(\%)$ & 2.9 & 5.8 & 0.0001 \\
\hline Red blood cells transfused $(\mathrm{ml})$ & $397 \pm 457$ & $554 \pm 824$ & 0.0001 \\
\hline Fresh frozen plasma transfused (ml) & $241 \pm 351$ & $314 \pm 591$ & 0.0100 \\
\hline
\end{tabular}

Values are presented as percentage occurrence for each group or as mean \pm standard deviation as appropriate.

Table IIA. Outcome variables found to occur significantly more frequently $(p<0.05)$ in patients with a primary diagnosis of sepsis as determined by multivariate analysis

\begin{tabular}{lccc}
\hline \multicolumn{1}{c}{ Variable } & Parameter estimate & Odds ratio & $p$ Value \\
\hline Seizures & 0.3685 & 1.446 & 0.0346 \\
Infarct or hemorrhage by head ultrasound & 0.8374 & 2.310 & 0.0001 \\
Other newrologic problem & 0.5926 & 1.809 & 0.0242 \\
Dialysis/hemofiltration & 0.3909 & 1.478 & 0.0131 \\
Symptomatic PDA & 0.5390 & 1.714 & 0.0190 \\
Culture-proven infection & 1.3950 & 4.035 & 0.0001 \\
Nat > 150 mEq/L & 0.7369 & 2.089 & 0.0019 \\
Hyperbilirubinemia & 0.8852 & 2.423 & 0.0001 \\
Dobutamine use & 0.6513 & 1.918 & 0.0001 \\
Bicarbonate use & 0.6027 & 1.827 & 0.0001 \\
Surfactant use & 0.4941 & 1.639 & 0.0085 \\
\hline
\end{tabular}

Table IIB. Outcome variables found to occur significantly less frequently $(p<0.05)$ in patients with a primary diagnosis of sepsis as determined by multivariate analysis

\begin{tabular}{lccc}
\hline \multicolumn{1}{c}{ Variable } & Parameter estimate & Odds ratio & $p$ Value \\
\hline Lung recovery & -0.5148 & 0.598 & 0.0185 \\
Bleeding at other surgical site & -1.3237 & 0.266 & 0.0008 \\
Pneumothorax necessitating treatment & -0.8177 & 0.444 & 0.0092 \\
Other pulmonary problem & -1.0025 & 0.367 & 0.0120 \\
\hline
\end{tabular}

included higher birth weight $(p=0.001)$, absence of cardiopulmonary arrest before ECMO $(p=0.002)$, higher arterial $\mathrm{pH}$ before ECMO $(p=0.0001)$, and lower ventilator rate required before $\operatorname{ECMO}(p=$ $0.0016)$. Recovery of lung function was less frequent in the septic group (odds ratio 0.598, $p=0.0185$ ). Recovery was also predicted by birth weight, most recent arterial $\mathrm{pH}$, and previous ventilatory rate. By multivariate analysis, fewer complications were statistically more common in the septic group. However, neurologic, renal, and metabolic factors remained significant (see Table IIA). In addition to the outcome variables listed in Tables IIA and IIB, four dichotomous variables achieved borderline statistical significance. These factors, all of which occurred more frequently in group 1 , were serum creatinine greater than $1.5 \mathrm{mg} / \mathrm{dl}$ (parameter estimate 0.3049 , odds ratio $1.357, p=0.0771$ ), myocardial stunning (parameter estimate 0.3172 , odds ratio $1.373, p=0.0896)$, suspected secondary sepsis with elevated leukocyte count (parameter estimate 0.5684 , odds ratio $1.766, p=0.0672$ ), and use of epinephrine (parameter estimate 0.4204 , odds ratio $1.523, p=0.0527$ ). Two continuous outcome variables were greater in group 2 and reached borderline statistical significance: volume of red blood cells transfused $(p=0.0998)$ and duration of ECMO support $(p=0.0562)$.

\section{Discussion}

The application of ECMO for neonates with sepsis has historically been associated with de- 
creased survival and hemorrhagic complications. Kornhauser and associates ${ }^{10}$ reported no survivors treated with ECMO for neonatal group B streptococcal pneumonia, although survival after ECMO for other indications (meconium aspiration syndrome) was significantly greater. Despite this negative view, other reports have advocated the use of ECMO in the management of septic states. ${ }^{11-13}$ Hocker and colleagues ${ }^{12}$ presented a retrospective experience of patients with early-onset group B streptococcal sepsis and observed an $87 \%$ survival rate in hypotensive, infected patients treated with ECMO. Survival was lower in similar patients treated conservatively $(p=0.06)$. Overall, their experience with ECMO for sepsis was similar to that of neonates supported by ECMO for other reasons. Olson, Couch, and McDonald ${ }^{13}$ reported on 10 neonates with sepsis, with a survival from ECMO of $70 \%$. The most encouraging results have been reported by McCune and colleagues, ${ }^{11}$ who found a survival of $100 \%$ for 10 neonates treated with ECMO for sepsis. However, these infants did have complications, including significantly prolonged ventilatory support after ECMO and a greater prevalence of chronic lung disease (30\% versus $12 \%$ ). These small retrospective studies are limited and do not allow evaluation of the effect of pre-ECMO factors or direct comparison with other groups of patients treated by ECMO.

As a result, this study was undertaken to critically compare results of ECMO in the septic group with those in patients treated for other indications. To obtain a sufficient number of patients and to allow consideration of other potentially interacting variables, we obtained data from the ELSO Registry and subjected them to a multivariate analysis. According to this technique, no significant difference in survival was observed between infants treated for septic or nonseptic indications (odds ratio $=0.83$, $95 \%$ confidence limits 0.60 to $1.14, p=0.2480$ ). These data suggest that earlier concerns questioning the efficacy of this modality for septic conditions may be unwarranted. Of note, a univariate analysis of these same survival data suggested a markedly worse survival outcome for patients with sepsis $(p=$ 0.0002 ). This illustrates the importance of considering the interaction of other factors when addressing survival questions. Variables that significantly predicted better survival included higher birth weight, absence of cardiopulmonary arrest before ECMO, higher arterial $\mathrm{pH}$ before ECMO, and lower ventilator rate required before ECMO.
In contradistinction to other reports, ${ }^{11}$ generalized bleeding complications were not significantly different in the population with sepsis when compared with the infants without sepsis undergoing ECMO in the ELSO Registry. In fact, bleeding at surgical sites was more common in the neonates without sepsis, a finding likely explained by the greater number of operative procedures being performed with ECMO in the nonseptic group., 14 Additionally, no significant difference in transfusion requirements for red blood cells, fresh frozen plasma, or platelets was observed. The causes of bleeding complications in patients undergoing ECMO are multifactorial and include heparin use, quantitative and qualitative platelet function changes induced by extracorporeal support, and shortened survival time of transfused blood products in patients undergoing ECMO ${ }^{15}$ These factors may be more important than the underlying disease process. However, thrombotic processes including consumptive coagulopathy may be more common in the neonates with sepsis. This factor may relate to the increased prevalence of intracranial hemorrhage and infarction seen in the infants with sepsis. More data would be required to determine the cause of this finding.

The septic group was found to have a significantly greater incidence of neurologic complications including seizures (odds ratio 1.446, 95\% confidence limits 1.027 to $2.034, p=0.0346$ ) and intracranial infarct or hemorrhage (odds ratio $2.310,95 \%$ confidence limits 1.679 to $3.180, p=0.0001$ ). McCune and colleagues ${ }^{11}$ also reported a higher incidence of intracranial hemorrhage in patients with sepsis as compared with other patients treated with ECMO ( $40 \%$ versus $26 \%$ ). To combat the risk of bleeding in this population, they altered their management scheme by reducing heparin doses and maintaining higher ECMO flows throughout the period of support. This strategy may reduce hemorrhage risk without increasing the risk of thrombus formation in the circuit. Thrombotic problems may manifest mainly in small vasculature, a factor that may contribute to the increased incidence of cerebral infarction found in the patients with sepsis. The present study does not allow analysis of these factors and does not differentiate between neurologic problems resulting from hemorrhage or thrombosis.

Lung recovery was found to be less likely in the patients with sepsis $(p=0.0185)$. This lower incidence of lung recovery in combination with similar survivals suggests that deaths from causes other than 
lung failure were more prevalent in the nonseptic group. The severity of lung damage in the patients with sepsis may therefore be greater. McCune and associates $^{11}$ found pulmonary involvement to be more severe in patients with sepsis and proposed this to be secondary to additional structural damage not seen with meconium aspiration syndrome or respiratory distress syndrome. In contrast, pulmonary hemorrhage was not different between groups, and pneumothorax necessitating treatment and other pulmonary problems were more common in the patients without sepsis. These latter phenomena do not seem to support the hypothesis that pulmonary damage is more severe in patients with sepsis. One may speculate that the pneumothorax complication may reflect the larger number of patients in the nonseptic group requiring ECMO for primary pulmonary processes such as congenital diaphragmatic hernia or air-leak syndrome. Further studies will be required to resolve the issue of the severity of lung disease in patients with sepsis.

An increased incidence of complications in other systems was noted in the septic group. The need for hemodialysis and hemofiltration was significantly greater in this group, with serum creatinine elevation following a similar trend. The requirement for nitroglycerin during ECMO was significantly greater in the patients with sepsis, implicating a greater incidence of hypertension. Hypernatremia was also more common in patients with sepsis, possibly reflecting renal damage. This renal injury may be related to the underlying septic state, the treatments used for the management of the sepsis, or the physiologic response to sepsis or extracorporeal support.

Study limitations. This study represents the largest collected series of patients with sepsis treated with ECMO. Nevertheless, several important limitations must be considered. The study is retrospective and spans a period of more than 6 years. ECMO practices and reporting patterns may have changed over this time period, and results will be biased toward centers that report consistently to this voluntary registry. As well, about $12 \%$ of each group carried a secondary diagnosis of sepsis, a factor not considered in the analysis, but one which may introduce a degree of uncertainty to the conclusions. Furthermore, registry data are incomplete in several important areas. Limited information was available regarding identification of specific causative organisms and management schema for sepsis (e.g., antibiotics, steroids) are not described. This information may be pertinent to the development of strategies to reduce the prevalence and severity of complications. Finally, because no long-term follow-up data are currently available from the registry, conclusions must be restricted to early outcomes only. Inasmuch as neurologic complications were a significant finding in the septic group, longer-term observation may be warranted. As in any new technology, the mechanics of ECMO are evolving. Although $89 \%$ of patients in this study underwent venoarterial ECMO, the current trend is toward increased use of venovenous ECMO support, which does not require carotid artery ligation. This may influence rates of neurologic or other complications. ${ }^{16}$

Although survival is not significantly worse in the septic population, strategies are needed to combat the variety of complications that are prominent in these patients. Close attention to coagulation indices may help to limit neurologic complications seen more commonly in the neonates with sepsis. Maintenance of precise fluid balance may help to limit renal damage and the need for hemodialysis or hemofiltration. This may also influence some of the other metabolic abnormalities. Further research into the host inflammatory response to extracorporeal circulation may yield other approaches that may limit or expand on these phenomena. Strategies capable of reducing both hemorrhagic and thrombotic complications would appear to be particularly useful in this subset of patients.

The progression of sepsis in the neonate is often resistant to conventional medical management. In contrast to earlier studies, cardiopulmonary support in the form of ECMO offers acceptable survival rates. Our analysis has demonstrated that a primary indication of sepsis does not independently predict a lower survival from ECMO when factors such as birth weight, cardiac arrest, and ventilator parameters are considered. Complication rates are higher in patients with sepsis as a primary indication for ECMO. In particular, neurologic events, renal deterioration, and metabolic problems occur more frequently. Bleeding complications are not found more frequently in neonates with sepsis. On the basis of these data, ECMO should not be withheld from neonates solely on the basis of sepsis. Rather, management strategies should focus on limiting the prevalence or severity of the common complications.

This study emphasizes the value of cooperative data registries in evaluation of new technology. Pooled data from multiple centers placed into a single database allow sufficient numbers for analysis of complex questions where multiple variables need 
to be considered. These registries can provide insight that any individual center is unlikely to experience.

We are grateful to Tom Delosh and Charles J. H. Stolar, MD, of the Extracorporeal Life Support Organization for providing access to the ELSO Registry database and to Donald McIntire, $\mathrm{PhD}$, for reviewing the statistical analysis.

\section{REFERENCES}

1. Hess ML, Hastillo A, Greenfield LF. Spectrum of cardiovascular function during gram-negative sepsis. Prog Cardiovasc Dis 1981;23:279-81.

2. Shankavan S, Farooki ZQ, Desai R. Hemolytic streptococcal infection appearing as persistent fetal circulation. Am J Dis Child 1982;136:725-30.

3. Barefield ES, Hicks TP, Philips JB III. Thromboxane and pulmonary morphometry in the development of the pulmonary hypertensive response to group B streptococcus. Crit Care Med 1994;22:506-14.

4. Bartlett RH, Roloff DW, Cornell RG, et al. Extracorporeal circulation in neonatal respiratory failure: a prospective randomized study. Pediatrics 1985;76: 479-87.

5. Bartlett RH, Gazzaniga AB, Jefferies R, et al. Extracorporeal membrane oxygenation (ECMO) cardiopulmonary support in infancy. Trans Am Soc Artif Intern Organs 1976;22:80-8.

6. Bartlett RH, Gazzaniga AB, Toomasian $\mathrm{J}$, et al. Extracorporeal membrane oxygenation (ECMO) in neonatal respiratory failure. Ann Surg 1986;204:23645.

7. Toomasian JM, Snedecor SM, Cornell RG, et al. National experience with extracorporeal membrane oxygenation for newborn respiratory failure. ASAIO Trans 1988;34:140-7.

8. O'Rourke PP, Crone RK, Vacanti JP, et al. Extracorporeal membrane oxygenation and conventional medical therapy in neonates with persistent pulmonary hypertension of the newborn: a prospective randomized study. Pediatrics 1989;84:957-63.

9. Langham MR Jr, Krummel TM, Bartlett RH, et al. Mortality with extracorporeal membrane oxygenation following repair of congenital diaphragmatic hernia in 93 infants. J Pediatr Surg 1987;22:1150-4.

10. Kornhauser MS, Gilbert PL, Desai HJ, et al. The efficacy of extracorporeal membrane oxygenation (ECMO) in meconium aspiration syndrome and Group B Streptococcal pneumonia. Pediatr Res 1988; 23:414A.

11. McCune S, Short BL, Miller MK, et al. Extracorporeal membrane oxygenation therapy in neonates with septic shock. J Pediatr Surg 1990;25:479-82.

12. Hocker JR, Simpson PM, Rabalais GP, et al. Extracorporeal membrane oxygenation and early-onset Group B Streptococcal sepsis. Pediatrics 1992;89:1-4.
13. Olson GL, Couch CL, McDonald JV. ECMO experience with septic newborns. Sixth Annual Children's Hospital National Medical Center ECMO Symposium. Breckenridge, Colo.: February 1990:A17.

14. Zwischenberger JB, Nguyen TT, Upp JR Jr, et al. Complications of neonatal extracorporeal membrane oxygenation: collective experience from the extracorporeal life support organization. J THORAC CARDIOVASC SURG 1994;107:838-49.

15. Robinson TM, Kickler TS, Walker LK, et al. Effect of extracorporeal membrane oxygenation on platelets in newborns. Crit Care Med 1993;21:1029-34.

16. Cornish JD, Heiss KF, Clark RH, et al. Efficacy of venovenous extracorporeal membrane oxygenation for neonates with respiratory and circulatory compromise. J Pediatr 1993;122:105-9.

\section{Discussion}

Dr. Julie A. Swain (Las Vegas, Nev.). This report is a valuable contribution. Adults obviously do not have meconium aspiration, so the indication for ECMO is far more often sepsis. Do you have any anecdotal data from your institution on the results of sepsis and ECMO in adults? I know those studies are in progress.

Dr. Meyer. Our data in the neonatal group have prompted us to initiate a review of sepsis in pediatric ECMO. It is likely that sepsis represents a larger problem in this group and that the results in the pediatric population may more closely relate to the adult experience, which is much smaller. Sepsis (especially pneumonia) is the most common primary indication for ECMO in pediatric cases, and overall survival rates are much lower than in neonates. We have also observed that patients in whom sepsis develops during ECMO have a significant decrease in survival and an increase in bleeding complications.

Dr. Tom Karl (Melbourne, Australia). We would agree that sepsis should not preclude candidacy for ECMO in either the neonate or the older patient. However, especially in the neonatal group, there is a fine line between primary sepsis, pulmonary hypertension, meconium aspiration, and other conditions for which a baby might require ECMO. How can one say that the patient's primary problem is sepsis, and how did you do it for this study?

Dr. Meyer. The precision of the primary diagnosis is important to the results of this retrospective registry analysis. The registry database contains a field for this variable that was recorded in all but four of the 6856 cases. The primary diagnosis field is rather general, and frequently in patients with sepsis the organism responsible is not recorded. The study is dependent on the accuracy of the ELSO Registry database, but so far as we can determine the primary diagnosis seemed well defined.

Dr. Karl. You would not know, for example, how many of these patients were not considered to have sepsis as a primary diagnosis but were receiving broad-spectrum antibiotics at the time that ECMO was initiated.

Dr. Meyer. The primary diagnosis was recorded for virtually all patients in the ELSO Registry and selected among the choices including meconium aspiration syndrome, congenital diaphragmatic hernia with persistent 
fetal circulation, sepsis, primary pulmonary hypertension of the newborn, respiratory distress syndrome, and other processes. These diagnoses are generally specific and exclusive. For this study, we assumed that clinicians focused on the recorded indication as their reason for initiating ECMO. The database also included a field for secondary indications, but these were not applied to this analysis.

Dr. Ralph Delius (Iowa City, Iowa). Do you have any information on the anticoagulation required in these patients? When I was in Ann Arbor we noted that the patients with sepsis usually had a coagulopathy. Anticoagulation had to be handled carefully to avoid bleeding problems.

With the greater use of the double-lumen cannula, do you have any information on venovenous ECMO compared with venoarterial ECMO?

Dr. Meyer. Anticoagulation strategies were not specifically recorded in the ELSO database. Activated clotted times were not available to us for this analysis. However, we did not find any significant differences in bleeding complications per se between the two groups, after controlling for standard pre-ECMO variables.

To address the second question, we did not stratify patients according to the technique of cannulation. The registry data analyzed were complete up to June 1993, with the majority of patients during this period (89\%) undergoing ECMO via a venoarterial approach. Since that time, there has been a proliferation of the use of venovenous techniques with double-lumen cannulas, but our information with this technology is limited. A potential advantage may be a reduction in the neurologic complications, because the carotid artery is not cannulated. However, thrombotic complications may become more prevalent unless higher flow rates are maintained in the system.

Dr. R. Siewers (Pittsburgh, Pa.). It is important to make a distinction between ECMO support for neonatal sepsis and ECMO support for pediatric sepsis. The relatively encouraging results with ECMO support in the neonatal group, greater than $75 \%$ survival, has not been matched in the older pediatric group, which has shown survivals in the $40 \%$ to $50 \%$ range as documented by the ELSO experience. At the Children's Hospital of Pittsburgh, we have some serious reservations about application of ECMO support in the pediatric age group, distinct from the neonatal group, when children are referred for respiratory insufficiency on the primary basis of sepsis. I am pleased to see that the wealth of data accumulated by ELSO is beginning to be presented and discussed in this forum.

Dr. Meyer. As I indicated, we are now in the process of reviewing data in the pediatric age group, where viral pneumonia is a more common problem. Survival rates appear to be worse in this subpopulation.

In the neonates, results of ECMO for sepsis are better, but the underlying cause is also different. From the minority of patients with organism data available in the registry, $74 \%$ of neonates placed on ECMO for sepsis had group B streptococcal infections. This disease process causes physiologic changes consistent with primary pulmonary hypertension of the newborn, and ECMO may be a more successful treatment modality for this disease.

\section{Appendix A: Outcome variables used in analysis}

\section{General outcome}

Survival

Recovery of lung function leading to discontinuation of ECMO

Duration of ECMO support

Mechanical complications

Oxygenator failure

Raceway rupture

Other tubing rupture

Pump malfunction

Heat exchanger malfunction

Clots in circuit

Air in circuit

Cracks in pigtails or connectors

Cannula placement or reposition problems

Restrictive sutures

Hemofilter malfunction

Kinking in cannula

Other mechanical problem

Hemorrhagic complications

Gastrointestinal hemorrhage necessitating transfusion

Bleeding at cannula site

Bleeding at other surgical site

Hemolysis

Other hemorrhagic problem

Neurologic complications

Brain death

Seizure

Infarction or hemorrhage by head ultrasound

Infarction or hemorrhage by computed tomography or magnetic resonance imaging

Other neurologic problem

Renal complications

Creatinine concentrations greater than $1.5 \mathrm{mg} / \mathrm{dl}$

Dialysis or hemofiltration

Other renal problem

Cardiopulmonary complications

Pulmonary hemorrhage

Cardiopulmonary resuscitation required

Myocardial stunning

Cardiac arrhythmias

Symptomatic patent ductus arteriosus

Hypertension necessitating vasodilator therapy

Other cardiopulmonary problem

Pneumothorax necessitating treatment

Other pulmonary problem

Septic complications

Culture-proven secondary infections

Secondary infection suspected by elevated white blood cell count

Other infectious problem

Transfusion requirements

Volume of packed red blood cells transfused

Volume of fresh frozen plasma transfused

Volume of platelets administered

Metabolic complications

Serum potassium level less than $2.5 \mathrm{mEq} / \mathrm{L}$ 
Serum potassium level greater than $6.0 \mathrm{mEg} / \mathrm{L}$

Serum sodium level less than $125 \mathrm{mEq} / \mathrm{L}$

Serum sodium level greater than $150 \mathrm{mEq} / \mathrm{L}$

Serum calcium level less than $6 \mathrm{mg} / \mathrm{dl}$

Serum calcium level greater than $12 \mathrm{mg} / \mathrm{dl}$

Blood glucose level less than $40 \mathrm{mg} / \mathrm{dl}$

Blood glucose level greater than $240 \mathrm{mg} / \mathrm{dl}$

Other blood sugar problems

$\mathrm{pH}$ less than 7.20

$\mathrm{pH}$ greater than 7.60

Hyperbilirubinemia

Other metabolic problems

Drug requirements

Dopamine administered

Dobutamine administered

Tolazoline administered

Nitroprusside administered

Isoproterenol administered

Prostaglandin E administered

Epinephrine administered

Nitroglycerin administered

Other vasoactive drug administered

Surfactant administered

Bicarbonate administered

\section{Appendix B: Pre-ECMO variables used in} evaluation

General

1. Patient age at time of ECMO

2. Patient sex

3. Birth weight

4. Cardiopulmonary arrest before ECMO

Most recent arterial blood gas before ECMO

5. $\mathrm{pH}$

6. Carbon dioxide tension

7. Oxygen tension

8. Bicarbonate

9. Saturation

Most recent ventilator settings before ECMO

10. Ventilator rate

11. Inspired oxygen fraction

12. Peak inspiratory pressure

13. Positive end-expiratory pressure level

14. Mean airway pressure

15. Oxygenation index (inspired oxygen fraction $x$ mean airway pressure $\times 100$ /arterial oxygen tension) 